\title{
Desarrollo y validación de un método analítico para la determinación de venlafaxina en suero mediante HPLC-UV
}

\author{
Developing and validating on HPLC-UV method for enlafaxine \\ determination in serum
}

\section{Desenvolvimento e validação de método analitico para determinação de da venlafaxina em soro empregando HPLC-UV}

\section{Luz A. Sarmiento-Rubiano ${ }^{1}$, Meibys P. Espinosa-Mejia ${ }^{2}$}

\author{
Bacterióloga, PhD, Tecnología de Alimentos \\ 2 Bacterióloga. Laboratorio de Cromatografía Universidad Metropolitana de Barranquilla - Fundación Hospital \\ Universitario Metropolitano. Email: lusarru@hotmail.com
}

Recibido: marzo 6 de 2012

Aceptado: octubre 1 de 2012

\begin{abstract}
Resumen
La Venlafaxina es un fármaco antidepresivo de la familia de los derivados sintéticos de las fenetilaminas bicíclicas cuya actividad consiste en inhibir la recaptación de serotonina, noradrenalina y en menor proporción dopamina en la neurona presináptica, potenciando la neurotransmisión a nivel del sistema nervioso central. Diversos métodos han sido reportados para el análisis de Venlafaxina en variados fluidos biológicos, siendo la cromatografía liquida de alta precisión HPLC uno de los más utilizados. En este estudio se ha desarrollado un método rápido y sencillo para la determinación de Venlafaxina en muestras de suero con extracción liquido-liquido, detección ultravioleta a $229 \mathrm{~nm}$, fase móvil isocrática y Carbamazepina como estándar interno, el rango de detección es entre 0,1 a $10 \mu \mathrm{g} / \mathrm{ml}$ con precisión y exactitud del 95\% y un tiempo total de elución de 25 minutos por muestra, el método es lo suficientemente sensible, exacto, y reproductible para ser usado en estudios farmacocinéticos.
\end{abstract}

Palabras clave. HPLC, Venlafaxina, antidepresivos, farmacocinética

\begin{abstract}
Venlafaxine is an antidepressant drug; it is asynthetic phenethylamine bicyclic derivative having antidepressant activity which consists of inhibiting serotonin, nor epinephrine and (to a lesser extent) dopamine reuptake in the presynaptic neuron, thereby promoting synaptic transmission in the central nervous system. Different methods have been reported for analysing venlafaxine in biological fluids, high-performance liquid chromatography (HPLC) being one of the most used to date. This study involved developing a rapid and simple method for determining venlafaxine in serum samples using liquid/liquid extraction, $229 \mathrm{~nm}$ UV detection, isocratic mobile phase and carbamazepine as internal standard. Linear (detection) range was 0.1 to $10 \mu \mathrm{g} / \mathrm{ml}$ (95\% precision and accuracy) and 25 mintotal elution time per sample. This method was sufficiently sensitive, exact and reproducible for use in pharmacokinetic studies.
\end{abstract}

Key words: HPLC, venlafaxine, antidepressant, pharmacokinetic. 


\begin{abstract}
Resumo
Venlafaxina é um antidepressivo da família dos derivados sintéticos da bicíclico fenetilaminas e tem como objectivo inibir à recaptação da serotonina, noradrenalina e em menor proporção a dopamina no neurônio pré-sináptico, e a melhoria da neuro transmissão no sistema nervoso central. Há registados vários métodos para o análise da venlafaxina nos fluidos biológicos, um dos mais utilizados é cromatografia líquida de alta presição (HPLC). Neste estudo foi desenvolvido um método simples e rápido para determinação da venlafaxina em amostras de soro com extracção líquido-líquido, detecção UV a 229 nm, fase móvel isocrática e carbamazepina como padrão interno. O nivel de detecção situa-se entre 0,1 e 10 ug / ml, com precisão e exactidão de 95\% e um tempo de eluição total de 25 minutos por amostra, o método é bastante sensível, preciso e reprodutível para serem usado em estudos farmacocinéticos.
\end{abstract}

Palavras-chave. HPLC, venlafaxina, antidepressivos, farmacocinética.

\section{Introducción}

La Venlafaxina es un fármaco antidepresivo de la familia de las fenetilaminas, su nombre químico es 1-[2-(Dimetilamino)-1-(4-metoxifenil)etil]ciclohexanol, se emplea comúnmente en la industria farmacéutica como Clorhidrato de Venlafaxina cuya formula empírica es $\mathrm{C}_{17} \mathrm{H}_{27} \mathrm{NO}_{2} \mathrm{HCl}$, tiene un peso molecular de 313,8 gramos mol de los cuales 277.4 son Venlafaxina pura, es una sustancia cristalina blanquecina soluble a $572 \mathrm{mg} / \mathrm{ml}$ en $\mathrm{NaCl} 0,2 \mathrm{M}$. Su actividad consiste en inhibir la recaptación de serotonina, noradrenalina y en menor proporción dopamina en la neurona presináptica, potenciando la neurotransmisión a nivel del sistema nervioso central. La Venlafaxina no tiene actividad inhibidora de la monoaminooxidasa (IMAO) es por ello que a diferencia de otros antidepresivos triciclicos no tiene efectos secundarios tan importantes a nivel cardiovascular (Pacher, 2004), aunque su efecto antiplaquetario puede representar un nivel de riesgo que ha generado la restricción de su uso en algunos países (Taylor. 2010). La dosis recomendada de Venlafaxina es de $75 \mathrm{mg}$ al día en tres dosis, de ser necesario se puede incrementar hasta $150 \mathrm{mg} /$ día e incluso ha 225 $\mathrm{mg} /$ día con aumento en intervalos de $75 \mathrm{mg}$ cada cuatro días (Schatzberg 2000). Después de su consumo oral, aproximadamente el $92 \%$ de la Venlafaxina es absorbido alcanzando una biodisponibilidad absoluta del $42 \%$, el grado de unión a proteínas plasmáticas es del $30 \%$ y es metabolizada en el hígado siendo su principal metabolito la O-desmetilvenlafaxina, molécula con igual actividad biológica, aproximadamente a las 48 horas el $87 \%$ de la Venlafaxina y sus metabolitos son eliminados por orina (FDA, 2005).

Diversos métodos han sido reportados para el análisis de Venlafaxina en variados fluidos biológicos, siendo la cromatografía liquida de alta precisión HPLC uno de los más utilizados unido a diferentes métodos de detección, como fluorescencia (Mandrioli, 2007), ultravioleta (Samanidou, 2011; Matoga, 2001) o detección electroquímica (Blier et al 2007), esta técnica también ha sido utilizada en combinación con otras tecnologías como la espectrometría de masas LC-MS/MS con ionización electrospray (Kingbäck, 2011; He, 2005) o la cromatografía de gases (Mastrogianni, 2012). Respecto a los protocolos de extracción utilizados, la fase solida (SFE) es en muchos casos el método de elección (Clement, 1998). En este estudio se ha desarrollado un método rápido y sencillo para la determinación de Venlafaxina en muestras de suero para ser empleado en estudios farmacocinéticos y se ha validado de acuerdo a los parámetros establecidos por la Oficina de las Naciones Unidas (ONU, 2010) y la FDA Americana (U.S. FDA, 2001). El método propuesto utiliza una extracción liquido-liquido de la molécula de Venlafaxina con un solvente no polar en medio alcalino, detección ultravioleta a $229 \mathrm{~nm}$, fase móvil isocrática y Carbamazepina como estándar interno. La Carbamazepina es un fármaco antiepiléptico derivado tricíclico del iminostilbeno de muy escasa solubilidad en agua, soluble en metanol y similar estructuralmente a los antidepresivos tricíclicos, contrariamente a la Venlafaxina cuya estructura difiere de estos, la diferencia estructural entre las dos moléculas permite una adecuada separación cromatográfica (figura 1).

\section{Materiales y métodos}

\section{Reactivos y equipos}

Se utilizó estándar secundario de Venlafaxina clorhidrato de Genfar lote NVF-10002- (JM-01)-001 con humedad 0,5\% y pureza de 99,2\%; Carbamazepina presentación comercial en tabletas de 200 mg de laboratorios Bussie S.A.; acetonitrilo y metanol al 99,9\% grado HPLC de J.T. Baker; Fosfato de potasio monobásico $\mathrm{KH}_{2} \mathrm{PO}_{4}$, fosfato de potasio bibásico $\mathrm{K}_{2} \mathrm{HPO}_{4}$ y acido fosfórico $\mathrm{H}_{3} \mathrm{PO}_{4}$ fueron grado analítico de Merck. Los equipos utilizados fueron: centrífuga Mikro 200, agitador vortex RELAX top de Heidolph, microbalanza analítica Sartorius, concentrador Vacufuge plus de eppendorf y congelador a $-20{ }^{\circ} \mathrm{C}$ thermo scientific. 

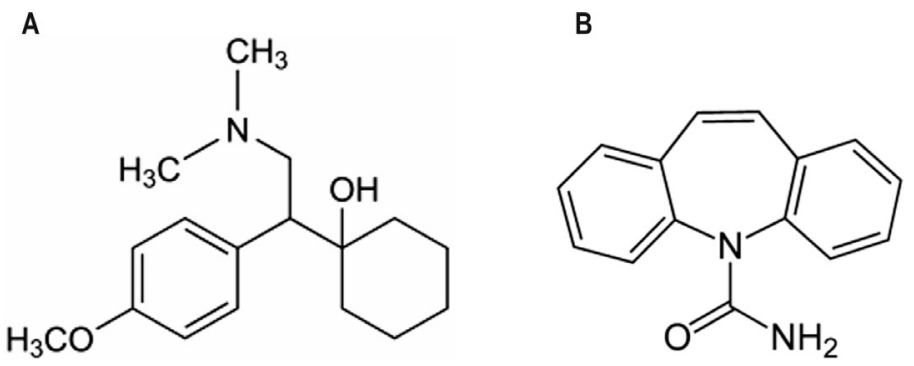

Figura 1. Estructura química de la Venlafaxina (A) y Carbamazepina (B).

\section{Sistema cromatográfico}

El equipo de cromatografía utilizado fué HPLC LaChrom Elite dotado de: Bomba L2130, Inyector L2200 y Detector UV L2400, Columna Hypersil GOLD C8

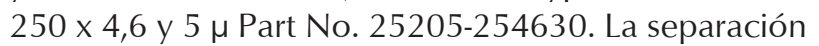
cromatográfica se realizó a temperatura ambiente. La fase móvil fué isocrática con flujo constante de 0,7 ml por minuto y consistió en acetonitrilo y buffer fosfato 0,062 M en una proporción 30:70 ajustado el pH a 5,5 con acido fosfórico. El tiempo de elución total fué de 25 minutos por muestra.

\section{Soluciones de trabajo}

Soluciones de trabajo de Venlafaxina clorhidrato y Carbamazepina a concentraciones de $1 \mathrm{mg} / \mathrm{ml}$ y 0,1 $\mathrm{mg} / \mathrm{ml}$ respectivamente, fueron preparadas en metanol al 99,9\% grado HPLC y colocadas inmediatamente a $-20^{\circ} \mathrm{C}$ hasta su uso.

\section{Sueros control}

Con firma de consentimiento informado se tomó una muestra de sangre (tres tubos sin anticoagulante) a 10 voluntarios sanos quienes manifestaron no estar tomando ningún medicamento, las muestras fueron centrifugadas a $900 \mathrm{~g}$ por minuto durante 10 minutos, los suero fueron extraídos y almacenados a $-20{ }^{\circ} \mathrm{C}$ hasta su uso. Casa suero de forma individual fué analizado en el HPLC para verificar la ausencia de picos interferentes con los analitos de interés y finalmente se realizo un pool de sueros a partir del cual se prepararon sueros control con concentraciones de Venlafaxina de $0.1,0.5,1,4,6$ y $10 \mu \mathrm{g} / \mathrm{ml}$ a partir la solución de Venlafaxina de $1 \mathrm{mg} / \mathrm{ml}$.

\section{Preparación de las muestras y curvas de calibración}

$400 \mu \mathrm{l}$ de suero control fueron colocados en un tubo eppendorf de 1,5 ml junto con $20 \mu \mathrm{l}$ de Carbamazepina de $0,1 \mathrm{mg} / \mathrm{ml}$ como estándar interno y $20 \mu \mathrm{l}$ de hi- dróxido de sodio $1 \mathrm{~N}$, se mezclo en vortex por 1 minuto y posteriormente se adiciono $1 \mathrm{ml}$ de dietil éter para la extracción de los analitos de interés haciendo una agitación en vortex durante 20 minutos, posteriormente la muestra fue centrifugada 10 min a 17.320 g por minuto y $0,5 \mathrm{ml}$ del éter fueron colocados en un nuevo eppendorf de 1,5 $\mathrm{ml}$ y evaporados ha sequedad en el concentrador Vacufuge plus de eppendorf a $30{ }^{\circ} \mathrm{C}$ y $450 \mathrm{~g}$ por minuto durante 25 minutos, el extracto seco fue reconstituido con $300 \mu \mathrm{l}$ de fase móvil, agitado en vortex durante 15 minutos y colocado en un vial para HPLC, $50 \mu \mathrm{l}$ del reconstituido fueron colocados en el automuestreador e inyectados en el HPLC. La curva de calibración se realizo procesando sueros control con concentraciones de Venlafaxina en un rango entre 0,1 a $10 \mu \mathrm{g} \mathrm{ml}-1$ (0.1, 0.5, 1.0, 4.0, 6.0 y $10 \mu \mathrm{g} \mathrm{ml-1)}$ por triplicado. Para determinar la linealidad del sistema, se estableció el valor de R2 en la curva de calibración y el coeficiente de variación entre las mediciones de una misma concentración.

\section{Capacidad de recuperación}

Soluciones de Venlafaxina de 0,1 y $10 \mu \mathrm{g} / \mathrm{ml}$ y sueros control con iguales concentraciones fueron analizados por triplicado para comparar los valores obtenidos en cada caso y se calculó el porcentaje de recuperación del analito.

\section{Especificidad}

La especificidad es la capacidad del método para diferenciar el analito en presencia de otros componentes de la muestra, debe determinarse en un nivel bajo de concentración. Diez muestras de suero provenientes de diferentes donantes fueron analizadas para verificar la ausencia de picos en el cromatograma interferentes con el analito en cuestión, posteriormente los cromatogramas fueron comparados con los de sueros control con concentraciones de Venlafaxina de 0,1 y 10 
$\mu \mathrm{g} / \mathrm{ml}$ adicionados de Carbamazepina como estándar interno.

\section{Precisión y exactitud}

Describe el grado de dispersión del valor obtenido respecto al valor real o conocido bajo las condiciones establecidas de análisis. Sueros control con concentraciones de Venlafaxina de $0.1,4$ y $10 \mu \mathrm{g} / \mathrm{ml}$ fueron analizados por triplicado y durante dos días diferentes para evaluar la repetitividad y reproducibilidad del sistema.

\section{Estabilidad de los analitos}

La estabilidad de los analitos se evaluó por triplicado en sueros control a concentraciones de Venlafaxina de 1,4 y $10 \mathrm{ug} / \mathrm{ml}$ y adicionados de $20 \mu \mathrm{l}$ de Carbamazepina de $0,1 \mathrm{mg} / \mathrm{ml}$ bajo las siguientes condiciones:

Estabilidad de congelamiento y descongelamiento: Los sueros descritos fueros sometidos a periodos de congelación a $-20^{\circ} \mathrm{C}$, descongelados a las 24 y 72 horas, procesados, evaluados y los resultados comparados con sueros sin congelar.

Estabilidad de los sueros control a temperatura ambiente: Los sueros control descritos fueron analizados después de ser procesados y permanecer 24 y 72 horas a temperatura ambiente en el automuestreador.

Estabilidad de las soluciones Stock: Soluciones stock de Venlafaxina de $10 \mu \mathrm{g} / \mathrm{ml}$ y de Carbamazepina de
$0,1 \mathrm{mg} / \mathrm{ml}$ fueron analizadas después de permanecer 24 y 72 horas a temperatura ambiente en el automuestreador.

\section{Resultados}

Se desarrolló y validó un método analítico mediante HPLC-UV para la determinación de Venlafaxina en suero humano con estándar interno Carbamazepina del cual se resumen las condiciones instrumentales en la tabla 1.

Los resultados obtenidos en los ensayos de especificidad muestran que en los cromatogramas de 10 sueros de donantes sanos no se observan picos interferentes con los analitos de interés, cuando sueros adicionados con Venlafaxina a concentraciones de 0,1 y $10 \mu \mathrm{g} / \mathrm{ml}$ y Carbamazepina de $0,1 \mathrm{mg} / \mathrm{ml} 20 \mu \mathrm{l}$ fueron analizados se observó la presencia de picos en el cromatográma correspondientes a estas dos sustancias en los tiempos de retención 9,1 min y 17,5 min respectivamente (figura 2).

Al realizar la curva de calibración de Venlafaxina (figura 3) con sueros control en concentraciones de 0.1, $0.5,1,4,6$ y $10 \mu \mathrm{g} / \mathrm{ml}$, los resultados del modelo lineal indican la correlación existente entre la concentración y la medida del área de los picos resultantes, se obtuvo un R2 estadístico de 0,999 y el coeficiente de variación entre las replicas de una misma concentración fue inferior al $2 \%$ en todos los casos, lo cual indica muy poca dispersión entre las mediciones (tabla 2).

Tabla 1. Condiciones instrumentales para la determinación de Venlafaxina en suero mediante HPLC-UV.

\begin{tabular}{l|c}
\multicolumn{1}{c|}{ Parámetros instrumentales } & Condiciones \\
\hline Fase móvil & Acetonitrilo, Buffer fosfato $0,062 \mathrm{M} 30: 70 \mathrm{pH}_{5,5, \mathrm{H}_{3} \mathrm{PO}_{4}}$ \\
Modo de elución & Isocrático \\
Longitud de onda & $229 \mathrm{~nm}$ \\
Columna & Fase reversa C8 $\mu \mathrm{m}, 250 \times 4,6 \mathrm{~mm}$ \\
Temperatura columna & Ambiente \\
Temperatura del automuestreador & Ambiente \\
Flujo de fase móvil & $0,7 \mathrm{ml} / \mathrm{min}$ \\
Volumen de inyección & $50 \mu \mathrm{L}$ \\
Modo de cuantificación & Estándar interno y curva de calibración \\
Modo de cualificación & Tiempo de retención \\
Unidades de concentración & $\mu \mathrm{gg} / \mathrm{mL}$ \\
Tiempo de retención Venlafaxina & $9,1 \mathrm{~min}$ \\
Tiempo de retención Carbamazepina & $17,5 \mathrm{~min}$ \\
Tiempo de corrida & $25 \mathrm{~min}$ \\
Volumen de muestra utilizado & $400 \mu \mathrm{L}$ \\
\hline
\end{tabular}



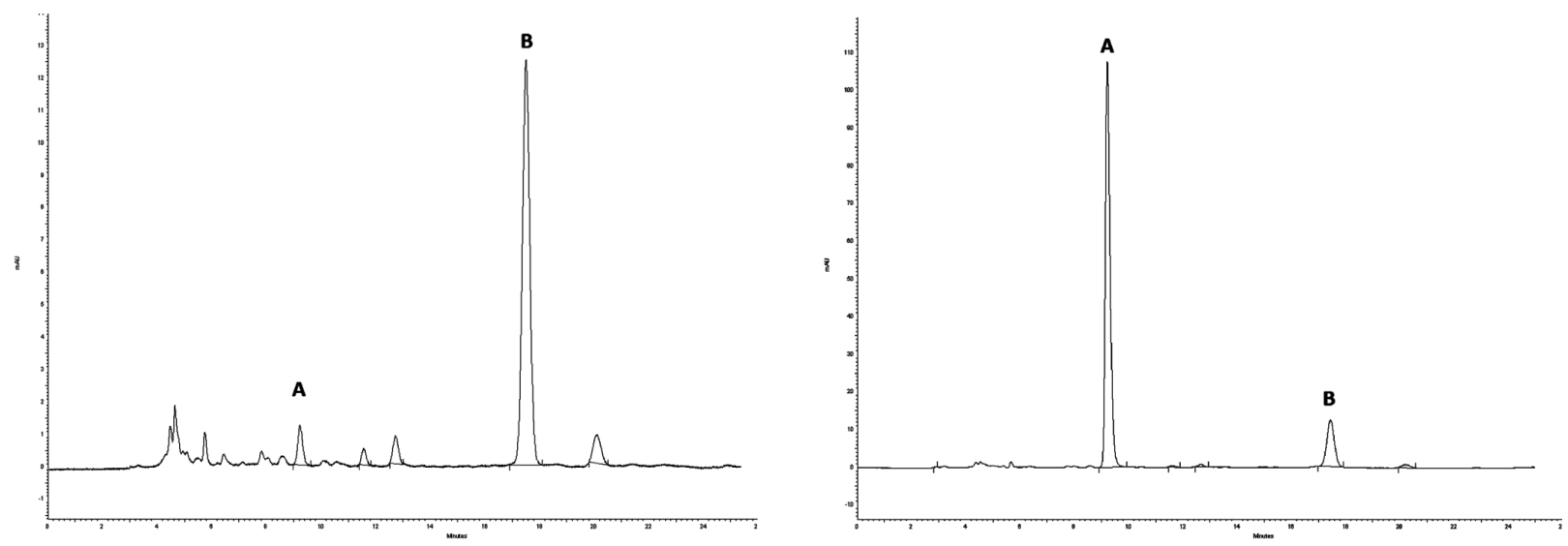

Figura 2. Cromatográma de Venlafaxina y Carbamazepina. (A) Venlafaxina (9,1 min) izquierda $0,1 \mu \mathrm{g} / \mathrm{ml}$, derecha $10 \mu \mathrm{g} / \mathrm{ml}(\mathrm{B})$; Carbamazepina 17,5 min.

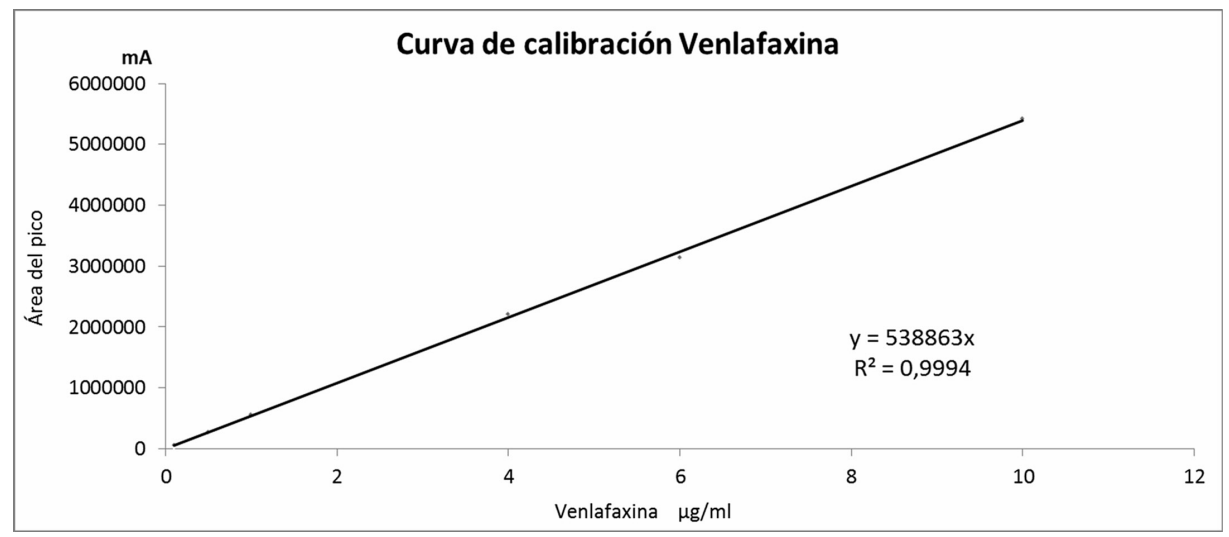

Figura 3. Curva de calibración de Venlafaxina con un rango de cuantificación entre 0,1 y $10 \mu \mathrm{g} / \mathrm{ml}$

La eficiencia de la extracción se calculo mediante la comparación de las relaciones de área de los picos de sueros control extraídos y las soluciones no extraídas observándose que a una concentración de Venlafaxina de $10 \mu \mathrm{g} / \mathrm{ml}$ la recuperación es de $98,74 \%$ y a concentración de 0,1 $\mathrm{\mu g} / \mathrm{ml}$ es de $98,69 \%$, el coeficiente de variación de las replicas en cada caso es inferior a $1 \%$ (tabla 3 ).

La precisión de las mediciones en las concentraciones de Venlafaxina $0.1,4$ y $10 \mu \mathrm{g} / \mathrm{ml}$ siempre tuvo un coeficiente de variación inferior a 1,5\%, respecto a la exactitud, el porcentaje de error de las mediciones a las concentraciones analizadas nunca fue superior a 3\%. (Tabla 4).

Los análisis de estabilidad muestran que la Venlafaxina en una muestra de suero es estable a la congelación y descongelación en los periodos analizados, cuando soluciones de Venlafaxina y sueros control con venlafaxina fueron dejados en el automuestreador por más de 24 horas perfectamente tapadas, se observo que no sufren alteración en su concentración.

\section{Discusion}

La cromatografía liquida de alta resolución es una técnica cada día mas utilizada para estudios farmacocinéticos, al tratarse de una tecnología sencilla, rápida, confiable, con costos relativamente bajos y una sensibilidad adecuada para el análisis en fluidos biológicos de muchos fármacos de uso común en humanos y animales. Para la identificación y cuantificación de Venlafaxina y Carbamazepina se selecciono la cromatografía liquida de fase reversa, que permitió una muy buena separación de los analitos gracias a sus diferencias de polaridad y su interacción con la fase estacionaria apolar de la columna C8 utilizada y la fase móvil de polaridad moderada. Respecto al manejo de la muestra, aunque la extracción en fase solida permite la recuperación de los analitos con el mínimo de interferencias, es un método que resulta más costoso 
Tabla 2. Resultados del estudio de linealidad.

\begin{tabular}{|c|c|c|c|c|}
\hline $\begin{array}{c}\text { Concentración } \\
\text { Venlafaxina } \\
\mu \mathrm{g} / \mathrm{ml}\end{array}$ & Área del pico & Promedio & $\begin{array}{c}\text { Desviación } \\
\text { estándar }\end{array}$ & $\begin{array}{c}\text { Coeficiente de } \\
\text { variación \% }\end{array}$ \\
\hline 10 & $\begin{array}{l}5382526 \\
5482844 \\
5402484\end{array}$ & 5422618 & 53103 & 0,98 \\
\hline 6 & $\begin{array}{l}3099120 \\
3206064 \\
3115121\end{array}$ & 3140102 & 57683 & 1,84 \\
\hline 4 & $\begin{array}{l}2160686 \\
2238236 \\
2221546\end{array}$ & 2206823 & 40818 & 1,85 \\
\hline 1 & $\begin{array}{l}543421 \\
564945 \\
552014\end{array}$ & 553460 & 10835 & 1,96 \\
\hline 0,5 & $\begin{array}{l}267715 \\
265026 \\
265487\end{array}$ & 266076 & 1438 & 0,54 \\
\hline 0,1 & $\begin{array}{l}54352 \\
54698 \\
55978\end{array}$ & 55009 & 857 & 1,56 \\
\hline
\end{tabular}

Tabla 3. Capacidad de recuperación de Venlafaxina en el proceso de extracción del suero.

\begin{tabular}{|c|c|c|c|c|c|}
\hline Concentración & $\begin{array}{l}\text { Solución de } \\
\text { Venlafaxina }\end{array}$ & $\begin{array}{l}\text { Suero con } \\
\text { Venlafaxina }\end{array}$ & \% recuperación & Promedio & $\begin{array}{c}\text { Coeficiente de } \\
\text { variación \% }\end{array}$ \\
\hline \multirow{3}{*}{10} & 5399782 & 5382526 & 99,680 & \multirow{3}{*}{98,735} & \multirow{3}{*}{0,857} \\
\hline & 5567879 & 5482844 & 98,473 & & \\
\hline & 5509876 & 5402484 & 98,051 & & \\
\hline \multirow{3}{*}{0,1} & 55098 & 54352 & 98,646 & \multirow{3}{*}{98,689} & \multirow{3}{*}{0,736} \\
\hline & 55823 & 54698 & 97,985 & & \\
\hline & 56296 & 55978 & 99,435 & & \\
\hline
\end{tabular}

y dispendioso cuando se pretende hacer un gran número de análisis, en el método desarrollado se utiliza la extracción liquido-liquido con un solvente no polar como el dietil éter en medio alcalino que permite una buena extracción de la Venlafaxina, la cual es posteriormente fácilmente recuperada por la rápida evaporación del éter. Mayoga et al en el 2001 describe una extracción de esta molécula con hexano- alcohol isoamílico en medio alcalino seguida de una solubilización de la Venlafaxina en medio acido, pero en este caso es posible que el extracto contenga mayor cantidad de impurezas apolares presentes en el suero que al realizar la extracción con éter.

Algunos métodos analíticos logran la diferenciación de la Venlaxina y su principal metabolito la O-desmetilvenlafaxina utilizando técnicas como HPLC acoplado a espectrometría de masas (Bhatt et al., 2005, Qin et 
Tabla 4. Resultados de la determinación de Venlafaxina en diferentes días.

\begin{tabular}{|c|c|c|c|c|c|}
\hline$\mu \mathrm{g} / \mathrm{ml}$ & $\begin{array}{l}\text { Suero con } \\
\text { Venlafaxina }\end{array}$ & $\begin{array}{c}\text { Área } \\
\text { del pico }\end{array}$ & $\begin{array}{c}\text { Venlafaxina } \\
\mu \mathrm{g} / \mathrm{ml}\end{array}$ & $\begin{array}{c}\text { Coeficiente } \\
\text { variación } \\
\% \\
\end{array}$ & $\begin{array}{c}\text { Error relativo } \\
\%\end{array}$ \\
\hline \multirow{6}{*}{10} & \multirow{4}{*}{ Día 1} & 5382526 & 9,990 & \multirow{6}{*}{0,625} & \multirow{6}{*}{0,665} \\
\hline & & 5482844 & 10,176 & & \\
\hline & & 5402484 & 10,027 & & \\
\hline & & 5428741 & 10,076 & & \\
\hline & \multirow[t]{2}{*}{ Día 5} & 5415565 & 10,051 & & \\
\hline & & 5419899 & 10,059 & & \\
\hline \multirow{6}{*}{4} & \multirow{4}{*}{ Día 1} & 2160686 & 4,008 & \multirow{6}{*}{1,400} & \multirow{6}{*}{2,749} \\
\hline & & 2238236 & 4,152 & & \\
\hline & & 2221546 & 4,121 & & \\
\hline & & 2198547 & 4,079 & & \\
\hline & \multirow[t]{2}{*}{ Día 5} & 2242512 & 4,160 & & \\
\hline & & 2230635 & 4,138 & & \\
\hline \multirow{6}{*}{0,1} & \multirow{4}{*}{ Día 1} & 54352 & 0,098 & \multirow{6}{*}{1,480} & \multirow{6}{*}{1,290} \\
\hline & & 54698 & 0,099 & & \\
\hline & & 55978 & 0,101 & & \\
\hline & & 56445 & 0,102 & & \\
\hline & \multirow[t]{2}{*}{ Día 5} & 55008 & 0,099 & & \\
\hline & & 55075 & 0,099 & & \\
\hline
\end{tabular}

al., 2010), la presencia de O-desmetilvenlafaxina se ha observado no solamente en muestras biológicas, sino también en formas farmacéuticas de Venlafaxina como producto de una degradación acida de la molécula (Carneiro et al 2010), aunque ambas moléculas son efectivas en el tratamiento de desordenes depresivos, difieren en su metabolismo ya que la Venlafaxina es oxidada por el citocromo p450 mientras que su metabolito no, cerca del 55\% de venlafaxina es eliminada por vía renal como O-desmetilvenlafaxina (Cohen. 2009). El método desarrollado en este trabajo, al igual que el reportado por Qin et al., 2008, se realizo con el fin de analizar solo la molécula de Venlafaxina en suero mas no sus metabolitos.

\section{Conclusiones}

El método analítico evaluado para la determinación de Venlafaxina en sangre utilizando como estándar interno Carbamazepina mediante la técnica HPLC-UV es especifico para el principio activo de interés, tiene la exactitud, precisión, linealidad y reproductividad adecuadas para dicho análisis, por lo tanto se puede utilizar en estudios de farmacocinética. Las condiciones cromatograficas utilizadas pueden permitir también la investigación de Venlafaxina en solución y pueden ser adaptadas y empleadas para el análisis cuantitativo en productos que contengan Venlafaxina como principio activo.

\section{Agradecimientos}

Al laboratorio de Toxicología Vargas-Melo de la Ciudad de Bogotá, por el aporte de algunos de los materiales y reactivos necesarios para la elaboración de este trabajo.

\section{Referencias}

Bhatt J, Jangid A, Venkatesh G, Subbaiah G, Singh S. Liquid chromatography-tandem mass spectrometry (LC-MS-MS) method for simultaneous determination of venlafaxine and its active metabolite O-desmethyl venlafaxine in human plasma. Journal of Chromatography B, 2005, 829 (1-2): 75-81.

Carneiro WJ, Andrade CH, Braga RC, Oliveira V. Identification of Desvenlafaxine, the major active metabolite of Venlafaxine, in in extended-release capsules. Revista electronica de farmacia. 2010;1: 39-53.

Clement EM, Odontiadis J, Franklin M. Simultaneous measurement of Venlafaxine and its major metabolite, oxydesmethylvenla- 
faxine, in human plasma by high-performance liquid chromatography with coulometric detection and utilisation of solid-phase extraction. Journal of Chromatography B: Biomedical Sciences and Applications, 1998; 705: 303-308

Cohen LJ. Desvenlafaxine: Frequently Asked Questions. Primary Psychiatry. 2009;16(12):1-8

Blier P, Saint-Andre E, Hdbert C. Effects of different doses of venlafaxine on serotonin and norepinephrine reuptake in healthy volunteers. International journal of neuropsychopharmacology. 2007;10(1): 41-50

FDA. Department of Health and Human Services Food and Drug Administration (2001). Center for Drug Evaluation and Research (CDER). Center for Veterinary Medicine (CVM). http://www. fda.gov/downloads/Drugs/GuidanceComplianceRegulatorylnformation/Guidances/ucm070107.pdf

FDA. Food and Drug Administration. (2005) Medication guide: about using antidepressants in children or teenagers. Rockville, MD. From the FDA website: http://www.accessdata.fda.gov/ drugsatfda_docs/label/2009/020151s054lbl.pdf

He J, Zhou Z, Li H. Simultaneous determination of fluoxetine, citalopram, paroxetine, venlafaxine in plasma by high performance liquid chromatography-electrospray ionization mass spectrometry (HPLC-MS/ESI). Journal of Chromatography B, 2005; 820: 33-39

Khan A, Zafar I, Jamshaid AK, Ghulam SK, Bilal M, Khan TM. The Development and Validation of HPLC-UV method for Analysis of Ciprofloxacin in serum and aqueous Humour. Archives of Pharmacy Practice. 2011;2(3):116-122

Kingbäcka M, Josefssonb M, Karlssona L, Ahlnera J, Bengtssona F, Fredrik C, Kugelberga CB. Stereoselective determination of Venlafaxine and its three demethylated metabolites in human plasma and whole blood by liquid chromatography with electrospray tandem mass spectrometric detection and solid phase extraction. Journal of Pharmaceutical and Biomedical Analysis. 2010;53: 583-590

Mandrioli R, Mercolini L, Cesta R, Fanali S, Amore M, Raggi M. Analysis of the second generation antidepressant venlafaxine and its main active metabolite O-desmethylvenlafaxine in hu- man plasma by HPLC with spectrofluorimetric detection. Journal of Chromatography B, 2007;856(1-2): 88-94

Mastrogianni O, Theodoridis G, Spagou K, Violante D, Henriques T, Pouliopoulos A, Psaroulis K, Tsoukali H, Raikos N. Determination of Venlafaxine in post-mortem whole blood by HS-SPME and GC-NPD Forensic Science International, 2011;215(1-3): 105-109

Matoga M, Pehourcq F, Titier K, Dumora F, Jarry C. Rapid high-performance liquid chromatographic measurement of Venlafaxine and O-desmethylvenlafaxine in human plasma Application to management of acute intoxications. Journal of Chromatography $\mathrm{B}, 2001 ; 760: 213-218$

ONU. Oficina de las Naciones Unidas contra la Droga y el Delito. (2010). Directrices para la validación de métodos analíticos y la calibración del equipo utilizado para el análisis de drogas ilícitas en materiales incautados y especímenes biológicos. http:// www.unodc.org/documents/scientific/Validation_Manual_STNAR41_Ebook_S.pdf

Pacher P, Kecskemeti V. Trends in the Development of New Antidepressants. Is there a Light at the End of the Tunnel?. Curr Med Chem. 2004;11(7): 925-943

Qin F, Li N, Qin T, Zhang Y, Li F. Simultaneous quantification of venlafaxine and O-desmethylvenlafaxine in human plasma by ultra performance liquid chromatography-tandem mass spectrometry and its application in a pharmacokinetic study. Journal of Chromatography B, 2010;878 (7-8):689-694

Qin XY, Meng J, Li XY, Zhou J, Sun X. Determination of venlafaxine in human plasma by high-performance liquid chromatography using cloud-point extraction and spectrofluorimetric detection. Journal of Chromatography B. 2008; 872 (1-2): 38-42

Samanidou V, Nazyropoulou C, Kovatsi L. A simple HPLC method for the simultaneous determination of Venlafaxine and its major metabolite O-desmethylvenlafaxine in human serum. Bioanalysis. 2011;3(15):1713-1718

Schatzberg Alan F. New indications for antidepressants. Journal of Clinical Psychiatry. 2000;61(11):9-17

Taylor David .2010. Venlafaxine and cardiovascular toxicity. BMJ Clinical Research (10). pp: 340:c411. 